\title{
High-precision gravity measurements using absolute and relative gravimeters at Mount Etna (Sicily, Italy)
}

\author{
Antonio Pistorio ${ }^{1,2,{ }^{\star}}$, Filippo Greco ${ }^{1}$, Gilda Currenti ${ }^{1}$, Rosalba Napoli ${ }^{1}$, Antonino Sicali ${ }^{1}$, \\ Ciro Del Negro ${ }^{1}$, Luigi Fortuna ${ }^{2}$ \\ ${ }^{1}$ Istituto Nazionale di Geofisica e Vulcanologia, Sezione di Catania, Osservatorio Etneo, Catania, Italy \\ ${ }^{2}$ Università di Catania, Dipartimento di Ingegneria Elettrica, Elettronica e Informatica (DIEEI), Catania, Italy
}

\author{
Article history \\ Received April 26, 2010; accepted July 26, 2011. \\ Subject classification: \\ Absolute and relative gravimeters, Uncertainty, Microgravimetry, Etna volcano, Volcano monitoring.
}

\begin{abstract}
Accurate detection of time gravity changes attributable to the dynamics of volcanoes requires high-precision gravity measurements. With the aim of improving the quality of data from the Mount Etna gravity network, we used both absolute and relative gravimeters in a hybrid method. In this report, some of the techniques for gravity surveys are reviewed, and the results related to each method are compared. We show how the total uncertainty estimated for the gravity measurements performed with this combined use of absolute and relative gravimeters is roughly comparable to that calculated when the measurements are acquired using only relative gravimeters (the traditional method). However, the data highlight how the hybrid approach improves the measurement capabilities for surveying the Mount Etna volcanic area. This approach enhances the accuracy of the data, and then of the four-dimensional surveying, which minimizes ambiguities inherent in the gravity measurements. As a case study, we refer to two gravity datasets acquired in 2005 and 2010 from the western part of the Etna volcano, which included five absolute and 13 relative stations of the Etna gravity network.
\end{abstract}

\section{Introduction}

High-precision gravity measurements can obtain the value of the acceleration of gravity with a precision of 1 $\mu \mathrm{Gal}\left(10^{-9} \mathrm{~g}\right.$; microgravimetry). They thus represent a useful tool for any volcano monitoring strategy, as they are essential for the detection of underground mass movements due to volcanic activity that might trigger a pre-eruptive state [Battaglia et al. 2003, Carbone et al. 2003, Hautmann et al. 2010, Greco et al. 2010, Williams-Jones and Rymer 2002]. Detection of clear gravity signals associated with the renewal of volcanic activity has led to increased applications for the four-dimensional gravity approach. Since the 1980s, we have used high-precision relative gravity measurements to determine significant correlations between the eruptive activity of the Mt. Etna volcano and temporal changes in the gravity field, which can occur with different patterns
[Budetta et al. 1999, Branca et al. 2003, Carbone et al. 2003, Bonforte et al. 2007, Carbone and Greco 2007, Greco et al. 2010, Bonaccorso et al. 2011].

Conventionally, gravity measurements have been carried out using relative spring gravimeters, which measure spatio-temporal gravity changes with respect to a fixed reference site. It is well known that the accuracy of these relative spring gravimeters is largely limited by instrumental drift. Together with influences arising from temperature and pressure variations, this instrumental effect can make the detection of volcanic source effects more difficult. This can hinder the detection of small time gravity changes that can be useful for an understanding of the geophysical processes preceding and accompanying volcanic unrest.

In the framework of a project with the Italian energy company Eni S.p.a., we had the opportunity to test a FG5\#238 absolute gravimeter from Micro-g LaCoste (owned by Eni S.p.a.) at Mt. Etna. As well as presenting here the performance of this instrument in a volcanic environment, we present the advantages of the use of a combined approach of absolute and relative gravimeter measurements performed at Mt. Etna volcano (hybrid gravimetry). The state of art clearly shows that the hybrid approach is a well-known advance and many applications have already been performed [Berrino 2000, Furuya et al. 2003, D'Agostino et al. 2008, Ferguson et al. 2008]. We demonstrate that the inclusion of repeated absolute stations within the existing gravity network for relative measurements represents an important step towards the improvement of the quality of the data acquired, which has obvious consequences for volcanic monitoring.

\section{Gravity network surveying Mt. Etna volcano}

The Mt. Etna gravity network was set up in 1986, and to reach its present configuration, it has been under continuous development over the intervening years. It consists of 71 


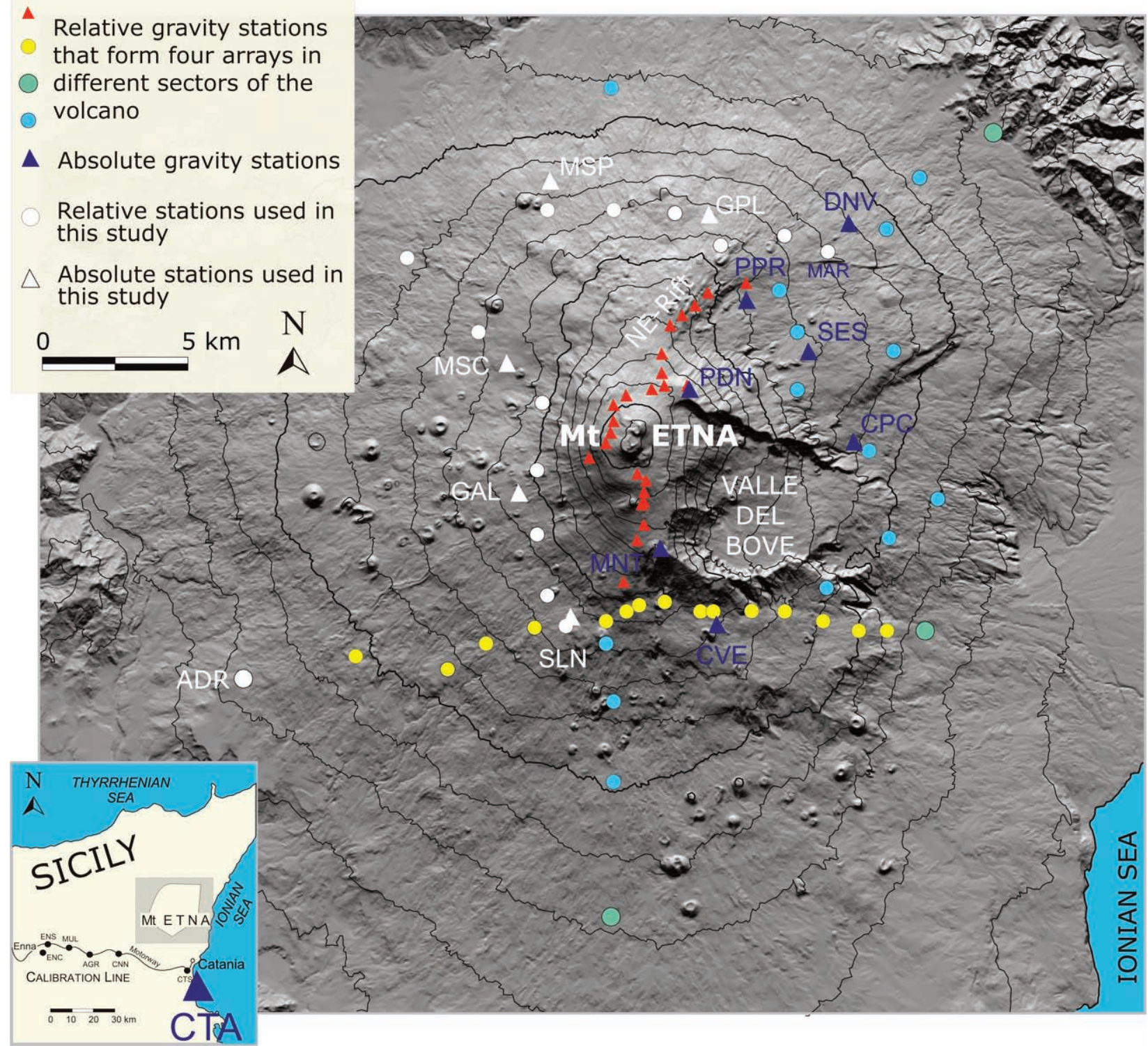

Figure 1. Sketch map of Mt Etna showing the current gravity network for absolute and relative measurements. White triangles and circles indicate, respectively, the absolute and relative stations in which the measurements reported in this study were made in 2005 and 2010 . At the bottom left, the calibration line for the relative gravimeters and the CTA absolute station are also indicated.

relative stations that are located between $0.5 \mathrm{~km}$ and $4 \mathrm{~km}$ apart, which cover an area of about $400 \mathrm{~km}^{2}$ and are arranged in four array subsets that are in circular profiles around the volcano and radial profiles along its slopes (Figure 1). These subarrays refer to a reference station that is not affected by volcanically induced gravity changes, and they differ from one another in station density, access (determined by snow cover), and time required to collect the gravity measurements. Each subarray can be occupied independently, which optimizes the flexibility in the data collection, to accommodate variations in activity and accessibility of the volcano. Measurements over the entire Mt. Etna gravity network are generally conducted once a year, although some of the arrays are occupied more frequently. Since 1994, discrete gravity measurements have been performed using a CG-3M\#9310234 Scintrex gravimeter (Figure 2a). Even when this instrument is used under the unfavorable conditions encountered on Mt. Etna (e.g. rough unpaved roads, marked differences in altitude), it provides high-precision measurements due to its low sensitivity to both shocks and external temperature changes. A calibration line (see Figure 1) was also established in February 1995, to monitor the systematic variations in instrumental calibration factors with time [Budetta and Carbone 1997].

To better constrain the gravity measurements acquired with relative gravimeters, we also started to collect absolute gravity data. We combined the use of absolute and relative gravimeters as a hybrid method to extend the potential of gravity measurements for the surveying of the Mt. Etna volcanic area. At present, the gravity network for absolute measurements is composed of 13 stations that are distributed around the volcano edifice at altitudes ranging between $1,250 \mathrm{~m}$ and $2,900 \mathrm{~m}$ a.s.l. (Figure 1 ). 
In this study, we focus on two different gravity surveys that were carried out for the western part of the Mt. Etna volcano (Figure 1). The first survey was performed in 2005 at 13 stations of the gravity network, and only a relative gravimeter was used (Scintrex CG-3M\#9310234). The second survey was carried out in 2010, and as well as the same relative stations, five absolute stations were added, with measurements using a FG5\#238 gravimeter as the reference points for the relative gravimeter (the hybrid approach).

As the different array subsets of the Mt. Etna gravity network have approximately the same characteristics (e.g. nonasphalted roads, stations $2-5 \mathrm{~km}$ apart, large altitude differences between pairs of stations, volcanic noise), even if we consider a restricted number of absolute and relative stations in a single sector of the volcano, the data from this case study can be considered representative for the entire network.

\section{Absolute gravity data}

Absolute gravity meters are based on the reconstruction of the trajectory followed by a test body subjected to the gravity field in a vacuum chamber. The ballistic absolute FG5\#238 gravimeter (Figure 2b) uses the freely falling method, where an object is dropped downwards [Niebauer et al. 1995]. The motion of the free-falling test body with an optical reflector is measured using a Michelson interferometer. An optical-fiber-connected, frequency-stabilized, He-Ne laser (red light, $633 \mathrm{~nm}$ ) is used to illuminate the interferometer where the input laser beam is split into the test and reference beams.

The test beam bounces on the reflective test body, while the reference beam travels straight through the interferometer. These beams are recombined and their interference signals (fringes) are used to track the trajectory of the test body. The time intervals between the occurrence of each interference fringe are measured by the time interval measuring system, with a $\mathrm{Rb}$ oscillator as a reference. A model equation derived from the law of motion of freefalling objects is fitted to the data. After removing the geophysical corrections (due to the Earth tides, ocean loading, polar motions) and instrumental effects (e.g. diffraction correction), the free-fall acceleration at the observation point is evaluated as the mean of all of the freefall accelerations obtained for each drop of the test body during a measurement session.

With the FG5\#238 gravimeter, a total of 700 timeposition points are recorded over the $20-\mathrm{cm}$-length trajectory of each drop. Usually, the repetition rate of each drop is 10 $\mathrm{s}$, even if drops can be produced every $2 \mathrm{~s}$. Measurements typically consist of one or two sets per hour, with the mean of several sets (usually 12 to 48 ) providing a final gravity value.

The software supplied by the Micro-g LaCoste was used for the data acquisition and analysis. This software provides an immediate value for the local gravity; it also includes a full-featured post-processor that allows the data analysis procedures to be varied and that calculates the statistical uncertainty $\left(\delta_{\text {stat }}\right)$, as given by the standard deviations of the absolute gravity values obtained for each set $\left(\sigma_{\text {set }}\right)$ divided by the square root of the number of sets, $N_{\text {set }}$ :

$$
\delta_{\text {stat }}=\sigma_{\text {set }} / \sqrt{N_{\text {set }}}
$$

The total uncertainty $\left(\delta_{a b s}\right)$ for the final gravity value is given by:

$$
\delta_{a b s}=\sqrt{\delta_{s t a t}^{2}+\delta_{s y s}^{2}}
$$

where $\delta_{s y s}$ is the systematic uncertainty that is due to different components of the measurements that can be grouped into

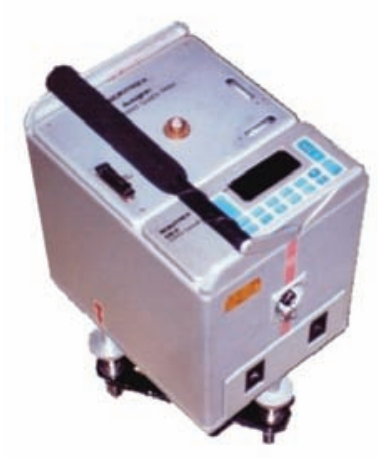

a

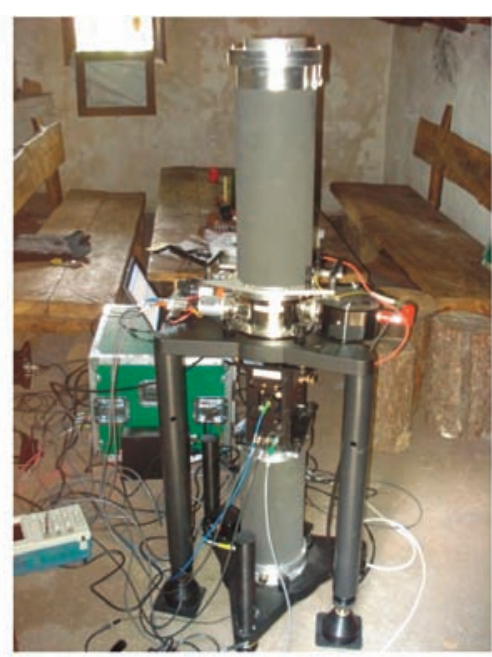

b

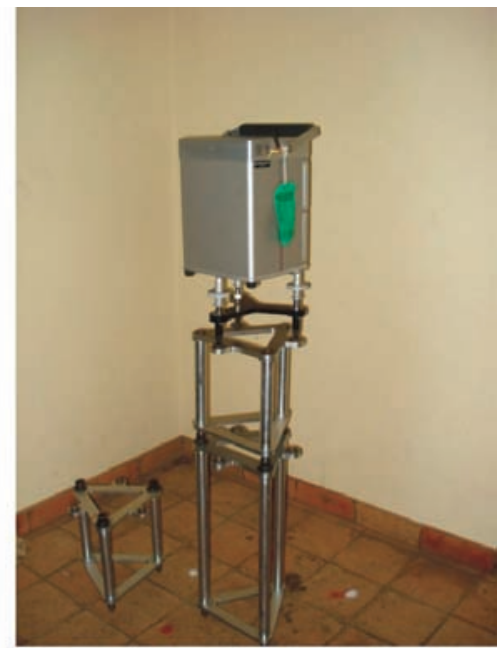

C

Figure 2. Gravimeters used at Mt. Etna volcano: (a) CG-3M\#9310234 Scintrex relative gravimeter; (b) FG5\#238 Micro-g LaCoste absolute gravimeter; (c) CG5\#8664.196 Scintrex relative gravimeter. 


\begin{tabular}{|c|c|c|c|c|c|c|c|}
\hline Station & $\begin{array}{l}\text { Latitude } \\
\left({ }^{\circ} \mathrm{N}\right)\end{array}$ & $\begin{array}{l}\text { Longitude } \\
\quad\left({ }^{\circ} \mathrm{E}\right)\end{array}$ & $\begin{array}{c}\text { Elevation } \\
\text { (m a.s.l.) }\end{array}$ & $\begin{array}{c}g_{\mathrm{abs}} \\
(\mathrm{mGal})\end{array}$ & $\begin{array}{c}\mathrm{h} \\
(\mathrm{mm})\end{array}$ & $\begin{array}{c}\delta \text { abs } \\
(\mu \mathrm{Gal})\end{array}$ & $\begin{array}{c}\mathrm{FAG} \pm \delta_{\mathrm{FAG}} \\
(\mu \mathrm{Gal} / \mathrm{m})\end{array}$ \\
\hline${ }^{\star} \mathrm{SLN}$ & 37.694 & 14.973 & 1730 & 979641363.5 & 1292.7 & 2.7 & $336.5 \pm 2.8$ \\
\hline${ }^{\star} \mathrm{GAL}$ & 37.732 & 14.950 & 1875 & 979600598.0 & 1293.7 & 7.5 & $279.6 \pm 3.3$ \\
\hline${ }^{\star} \mathrm{MSC}$ & 37.770 & 14.950 & 1720 & 979632850.7 & 1289.7 & 4.0 & $282.1 \pm 3.0$ \\
\hline${ }^{\star} \mathrm{MSP}$ & 37.823 & 14.961 & 1450 & 979693569.4 & 1291.7 & 3.0 & $297.5 \pm 2.2$ \\
\hline${ }^{\star} \mathrm{GPL}$ & 37.827 & 15.028 & 1570 & 979667690.6 & 1296.2 & 2.8 & $310.8 \pm 3.5$ \\
\hline
\end{tabular}

Table 1. Results of absolute gravity measurements acquired in 2010 using a FG5\#238 gravimeter. The first four columns give the acronyms and the coordinates of the absolute gravity stations (the locations of the sites are mapped in Figure 1 ). The last four columns give their measured gravity values $\left(g_{a b s}\right)$ referred to the height $(h)$, the total uncertainty $\left(\delta_{a b s}\right)$, and the free-air vertical gradient (FAG) with the uncertainty $\left(\delta_{F A G}\right)$. The symbol * indicates the absolute reference point.

four separate areas: (i) modeling; (ii) system; (iii) environment; and (iv) set-up. The systematic uncertainty of the FG5\#238 gravimeter is about $2 \mu \mathrm{Gal}$, as reported by Micro-g LaCoste.

The measurements of the absolute gravity with the total uncertainty $\left(\delta_{a b s}\right)$ gathered in 2010 in five selected stations of the Mt. Etna gravity network (Figure 1) are given in Table 1. Considering the difficulties of running such measurements under non-laboratory conditions and in an environment where the observation sites are affected by severe ambient conditions, the absolute gravity values (adjusted for earth tides, ocean loading, and local atmospheric and polar-motion effects) show a total uncertainty ranging from $2.7 \mu \mathrm{Gal}$ to 7.5 $\mu \mathrm{Gal}$ (see Table 1). Data scattering due to the strong floor vibrations, together with strong temperature and humidity fluctuations [Van Camp et al. 2005], were the most significant limitations to the uncertainty of the absolute gravity values measured at Mt. Etna.

\subsection{Test measurements using the FG5\#238 gravimeter}

The absolute data presented here refer to the stations located at altitudes between $1,400 \mathrm{~m}$ and $1,900 \mathrm{~m}$ a.s.l. At elevations higher than $2,000 \mathrm{~m}$ a.s.l. it was impossible to take the measurements using the FG5\#238 gravimeter because the laser beam was not generated. To determine the possible cause of this problem, various considerations were taken into account, including the power-supply system, and the humidity, temperature and pressure of the sites where the tests were carried out. On Mt. Etna we used the same gasoline generator at all of the absolute stations without any problems; therefore, we can exclude that the problem was related to the power generator. We also excluded the humidity, as we had measured $g$ at lower-altitude sites but under wetter conditions. For the ambient temperature, to ensure an operative temperature in the stations, we used a gas heater that faces the laser. Although the temperature had reached about $20{ }^{\circ} \mathrm{C}$, the FG5\#238 gravimeter still did not work. After these tests were performed, we hypothesized that the problem was due to the high altitudes of the sites (which correspond to low atmospheric pressures) where the FG5\#238 gravimeter was used.

Gravity measurements are affected by the simultaneous actions of various physical parameters that act both on the local gravity field and on the behavior of the gravimeter. A scheme of a generalized configuration that indicates the significant input-output relationships for all measuring apparatus is shown in Figure 3 [Doebelin 1990]. The input quantities are classified into three categories: the desired

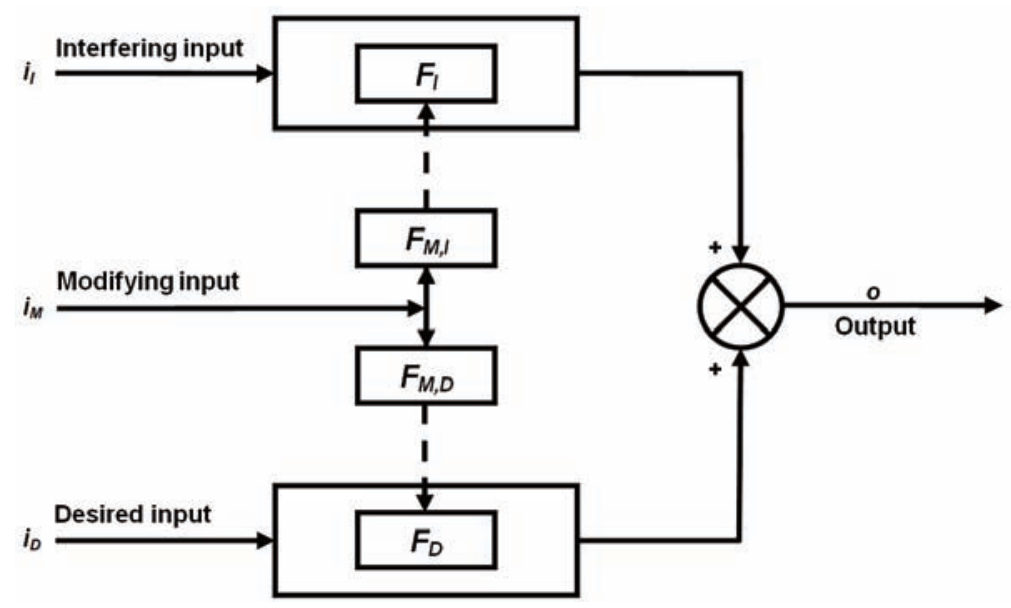

Figure 3. Scheme of a generalized configuration that highlights the significant input-output relationships present in all measuring apparatus (after Doebelin [1990]); see text for details. 


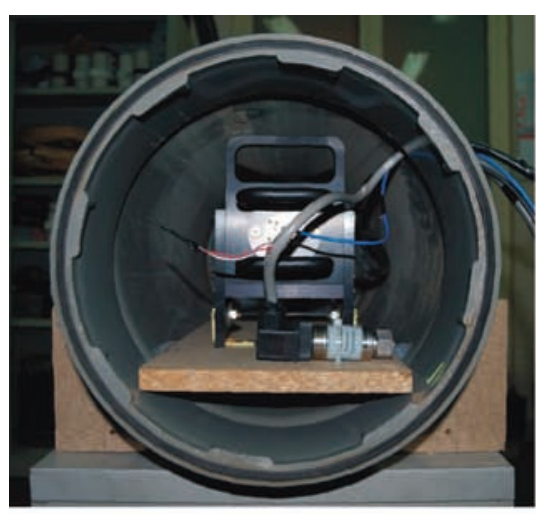

a

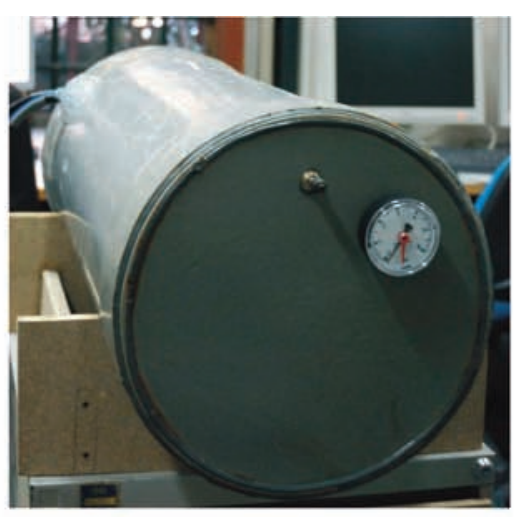

b

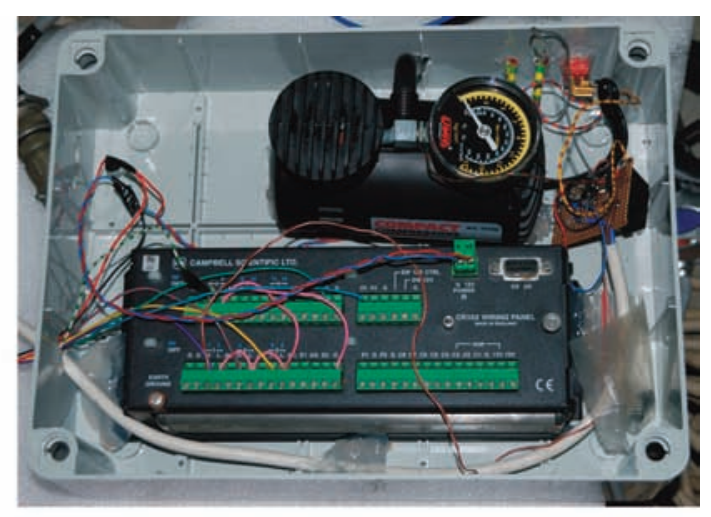

C

Figure 4. The hyperbaric chamber made from a PVC pipe. The laser, together with pressure (PTX 1400), temperature (LM 35) and humidity (HIH 3605) sensors, were placed inside the chamber $(\mathrm{a}, \mathrm{b})$. All of the signals were sampled at 1-min intervals and stored by a datalogger (CR10X). A $12 \mathrm{~V}$ compressor was installed for automatic adjustment around the operative pressure values threshold (c).

inputs, the interfering inputs, and the modifying inputs. The desired inputs represent the quantities that the instrument is specifically intended to measure. The interfering inputs represent the quantities to which the instrument is unintentionally sensitive. $F_{D}$ and $F_{I}$ are the symbolized inputoutput relations for these desired and interfering inputs, respectively. The modifying inputs are the quantities that cause a change in the input-output relations for the desired and interfering inputs. The symbols $F_{M, I}$ and $F_{M, D}$ represent the specific manners in which $i_{M}$ affects $F_{I}$ and $F_{D}$, respectively. Basically, for both absolute and relative gravimeters, the main interfering inputs are temperature and humidity, while the pressure is considered to be a modifying input (at least with modern gravimeters). Changes in atmospheric pressure can affect the gravity measurements in two ways: directly, through gravitational effects; and indirectly, although to a lesser extent, through surface deformation of the Earth due to the weight of the atmosphere [Warburton and Goodkind 1977]. The effects produced in measuring instruments that are not related to a gravity-field variation are considered to be negligible. However, for the FG5\#238 gravimeter, we assumed that the pressure is both a modifying input and an interfering input. To demonstrate this hypothesis, we tried to reproduce the same atmospheric-pressure conditions at the high altitude sites, such as in the Catania laboratory (50 $\mathrm{m}$ a.s.1.). For this, we developed a hyperbaric chamber where the laser was isolated (see Figure 4a,b). The hyperbaric chamber was obtained from a PVC pipe that was sealed at both ends, in which cavities were made to allow the passage of the cables and the optical fiber; all of the cavities were made watertight. In addition to the laser, an absolute pressure sensor (PTX 1400), a temperature sensor (LM 35), and a humidity sensor (HIH 3605) were also placed inside the chamber. The temperature was also monitored outside the chamber through another temperature sensor similar to that inside the chamber. All of the signals were sampled at 1-min intervals and stored by a datalogger (CR10X). The experiment was performed at the PDN observatory (2,800 $\mathrm{m}$ a.s.l.; see Figure 1). With the compression within the chamber, the pressure value increased from $707 \mathrm{mbar}$ (corresponding to the pressure at an altitude of $2,800 \mathrm{~m}$ a.s.1.) up to $900 \mathrm{mbar}$. At about 900 mbar, the laser suddenly began to work (see Figure 5). A threshold value for the pressure was determined as just above 900 mbar, and an automatic system using a Schmitt Trigger circuit (with a window $65 \mathrm{mbar}$ ) to manage a $12 \mathrm{~V}$ compressor (see Figure 4c) was installed for automatic adjustments around this pressure threshold. Using this unusual solution, we were able to take measures also at stations with altitudes greater than $2,000 \mathrm{~m}$ a.s.l.

This clearly showed a net dependence of the laser of the FG5\#238 absolute gravimeter on atmospheric pressure. Accordingly, a dependence between changes in the laser frequency and variations in the atmospheric pressure has already been shown [Chartier 1987].

We hypothesized that there were two reasons for this stopping of the generation of the laser at low atmospheric pressure that can be checked: it might have been a misalignment of the optical components of the laser, or a change in the refraction index of the air. Unfortunately, neither of these effects have been demonstrated previously.

\section{Relative gravity data}

The high-precision gravity measurements were carried out using relative gravimeters, which measure spatial changes with respect to a fixed reference site. Basically, with relative gravimeters, the gravity differences $(\Delta g)$ are measured between a pair of stations, and according to the study objectives and field conditions, the 'step method', 'star method' and/or 'profile method' can be used [Watermann 1957]. To measure the gravity network of Mt. Etna, the step method is mainly used. Following the multiple occupation sequence in the various stations, as required by this method (eg. A-B, B-A, A-B, B-C, ...), and starting from a primary reference point, each station was occupied at least three 


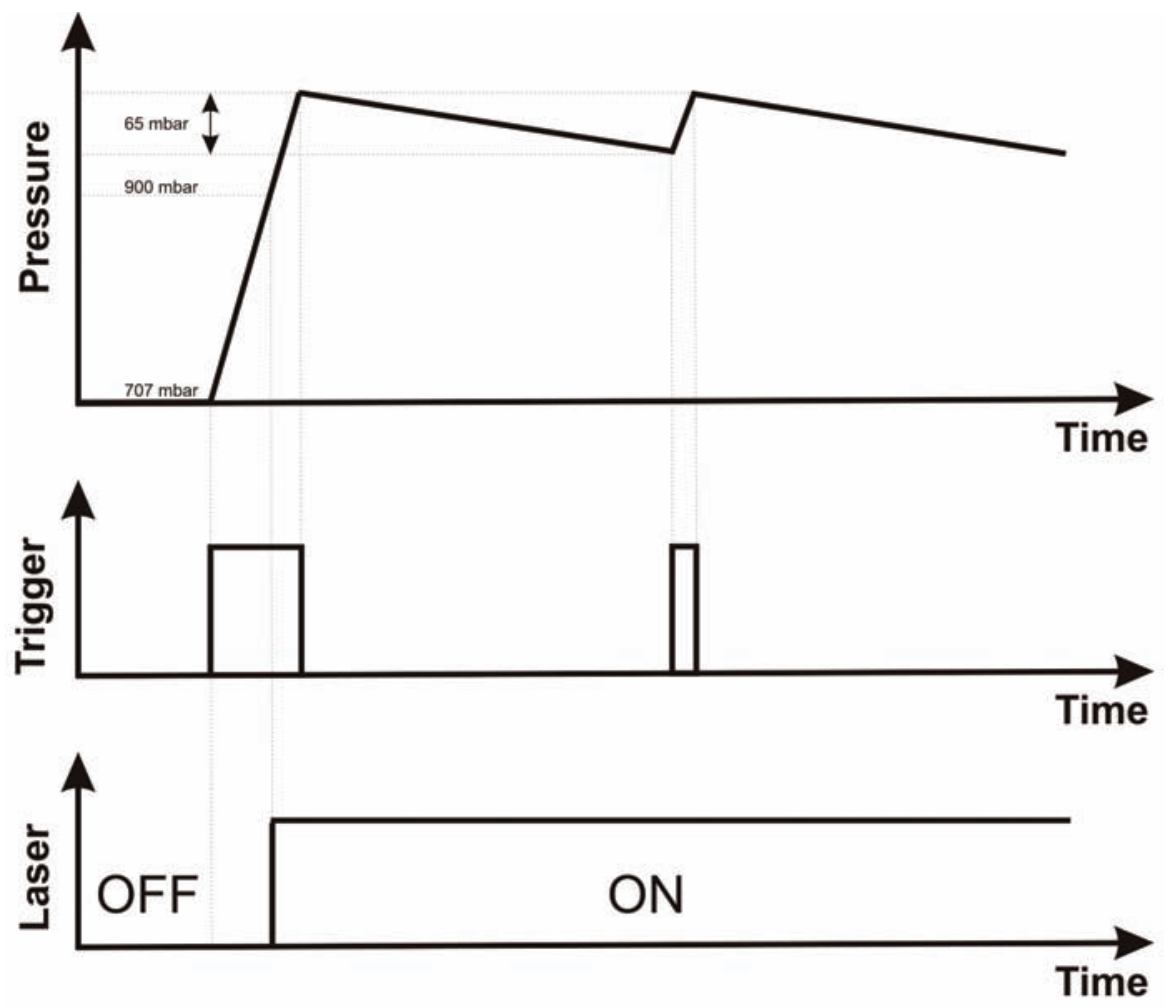

Figure 5. Waveforms of the internal pressure of the chamber (a), the signal to drive the $12 \mathrm{~V}$ compressor (b), and the activation of the laser (c).

times. In this way, the final $\Delta g$ values between the pairs of adjacent stations can be obtained from the mean of the three $\Delta g$ calculated, and the uncertainty of the link $\left(\delta_{\Delta g}\right)$ to assign to this value will be the square root of the sum of the squares of the individual uncertainties attributed to each $\Delta g$. Table 2 shows the $\Delta g$ with the uncertainty $\delta_{\Delta g}$ between the pairs of adjacent stations as collected in 2005 for the 13 selected stations of the relative gravity network (Figure 1). These were adjusted for tidal effects using a local-tide model, corrected for the instrumental drift, and referred to a single

\begin{tabular}{|c|c|c|c|c|c|}
\hline Station & Link & $\begin{array}{c}\Delta g \\
(\mathrm{mGal})\end{array}$ & $\begin{array}{c}\delta_{\Delta g} \\
(\mu \mathrm{Gal})\end{array}$ & $\begin{array}{c}g_{\mathrm{ADR}} \\
(\mathrm{mGal})\end{array}$ & $\underset{(\mu \mathrm{ral})}{\delta_{\text {rel }}}$ \\
\hline IFO & IFO-MDZ & -10.288 & 8 & 650.856 & 6 \\
\hline MDZ & MDZ-MFO & -32.148 & 10 & 640.568 & 7 \\
\hline $\mathrm{MFO}$ & MFO-MPA & -22.502 & 8 & 608.442 & 8 \\
\hline MPA & MPA-MNZ & 29.085 & 5 & 585.940 & 8 \\
\hline MNZ & MNZ-BCH & 32.148 & 10 & 615.025 & 8 \\
\hline $\mathrm{BCH}$ & BCH-RLN & 33.901 & 6 & 647.173 & 8 \\
\hline RLN & RLN-MSP & 12.925 & 6 & 681.074 & 8 \\
\hline MSP & MSP-L81 & 16.658 & 6 & 693.999 & 9 \\
\hline L81 & L81-MSM & -49.171 & 3 & 710.657 & 7 \\
\hline MSM & MSM-GLA & -25.376 & 7 & 661.486 & 7 \\
\hline GLA & GLA-GPA & 20.633 & 3 & 636.110 & 6 \\
\hline GPA & GPA-MRO & 13.474 & 3 & 656.743 & 9 \\
\hline MRO & MRO-MAR & 29.392 & 5 & 670.317 & 6 \\
\hline
\end{tabular}

Table 2. Results of the traditional gravity measurements acquired in 2005 for the 13 selected relative gravity stations of the Mt. Etna gravity network. The columns indicate, respectively, the station acronyms, the links between two adjacent relative stations, the gravity differences $(\Delta g)$ between each pair of adjacent relative stations; the uncertainties $\left(\delta_{\Delta g}\right)$ associated to each $\Delta g$; the gravity values $\left(g_{A D R}\right)$ at each station obtained with the least-squares method, as described in the text and referred to ADR station, and the associated errors $\left(\delta_{\text {rel }}\right)$. 
reference station (ADR; Figure 1). The distances between the adjacent stations ranges from $1 \mathrm{~km}$ to $3 \mathrm{~km}$, and each station can be reached on unpaved roads.

Considering all of the gravity stations as part of a loop, to evaluate the gravity and the associated total uncertainty at each station, we applied a strict compensation procedure that was based on a least squares adjustment method, by solving a linear system that consisted of observation equations (the method of indirect observations).

Errors in the gravity measurements can occur both for the measuring point and during the transfer from one station to another. Obviously, these errors will have an accidental and a systematic component. The errors that can accumulate at the measurement point are those that arise from incorrect readings or imperfect leveling of the instrument, or errors induced by the external environment (e.g. mechanical vibrations, microseisms, magnetic effects). Relevant effects are also caused by temperature gradients and pressure. During the transfer from one station to another, the mechanical vibrations that can cause changes to the behavior or properties of the gravimeters are particularly critical. The most important systematic errors originate between two stations; to eliminate this, observations are treated as gravity differences between pairs of stations occupied consecutively. The differences between the accidental errors of the two stations are considered to have a stochastic distribution.

The systematic components of the errors are included in the observation equation, which takes the general form of:

$$
\sqrt{P_{n}}\left(g_{i}-g_{j}-k_{m} \Delta g_{i j}\right)=\varepsilon_{i j}
$$

where $P_{n}$ is the observation weight, $g_{i}$ and $g_{j}$ are the unknown gravity values at the $i$-th and $j$-th stations, $k_{m}$ is the unknown scale factor for the $m$-th link, $\Delta g_{i j}$ is the measured gravity difference between the $i$-th and $j$-th stations, and $\varepsilon_{i j}$ is the residual. The solution with the least squares method for the linear system of observation equations, the number of which corresponds exactly to the measured gravity differences, leads to the adjusted $g$ values. To describe the mathematical procedure, considering the $P_{n}$ term of Equation (3) equal to 1 , the equation simplifies to:

$$
g_{i}-g_{j}-k_{m} \Delta g_{i j}=\varepsilon_{i j}
$$

and starting from the known terms $g_{i}^{0}, g_{j}^{0}$ and $k_{m}{ }^{0}$ :

$$
\begin{gathered}
g_{i}=g_{i}^{0}+x_{i} \\
g_{j}=g_{j}^{0}+x_{j} \\
k_{m}=k_{m}^{0}+x_{m}
\end{gathered}
$$

where $x_{i}, x_{j}$ and $x_{m}$ are the unknown corrections to be made to the known terms $g_{i}^{0}, g_{j}^{0}$ and $k_{m}^{0}$. Therefore Equation (4) becomes:

$$
\left(g_{i}^{0}+x_{i}\right)-\left(g_{j}^{0}+x_{j}\right)-\left(k_{m}^{0}+x_{m}\right) \Delta g_{i j}=\varepsilon_{i j}
$$

for the known terms $g_{i}^{0}, g_{j}^{0}$ and $k_{m}^{0}$ :

$$
g_{i}^{0}-g_{j}^{0}-k_{m}^{0} \Delta g_{i j}=l_{i j}
$$

Equation (4) can be further simplified as:

$$
x_{i}-x_{j}-x_{m} \Delta g_{i j}+l_{i j}=\varepsilon_{i j}
$$

or in matrix notation, as:

$$
A x+l=v
$$

where $A$ is the $n \times r$ matrix of the $r$ unknowns $\left(x_{i}, x_{j}\right.$ and $\left.x_{m}\right)$ due to the $n$ measured gravity difference: the coefficients of the matrix are +1 for $x_{i},-1$ for $x_{j}$, and $-\Delta g_{i j}$ for $x_{m} ; \boldsymbol{x}$ is the vector of the $r$ unknown corrections, $l$ is the vector of the $n$ errors calculated with the known terms $g_{i}^{0}, g_{j}^{0}$ and $k_{m}^{0}$, and $v$ is the vector of the $n$ residuals.

To satisfy the least-squares method, the following relationship must exist:

$$
\boldsymbol{v}^{\mathrm{T}} \boldsymbol{v}=\min
$$

and by defining the following matrix:

$$
R=A^{\mathrm{T}} \boldsymbol{A}
$$

the unknowns $x_{i}, x_{j}$ and $x_{m}$ are obtained by the expression:

$$
\boldsymbol{x}=\boldsymbol{R}^{-1} A^{\mathrm{T}} \boldsymbol{l}
$$

with the residuals $\varepsilon_{i j}$ :

$$
v=A x+l
$$

Therefore, through Equation (14), we can calculate the corrections to be included in Equations (5), (6) and (7), to obtain the values of the gravity. The determination of the gravity values at each station (see Table 2 ) is achieved through iterative methods: after each iteration, the new approximated values are calculated and used for the next iteration. All of the links are rejected where the residuals $\varepsilon_{i j}$ exceed the limit of $3 \sigma_{0}$, where $\sigma_{0}$ is the standard deviation of the unit weight. The value of $\sigma_{0}$ is related to the theoretical variance of the unit weight $\sigma_{0}^{2}$ from the equation:

$$
\sigma_{0}=\sqrt{\sigma_{0}^{2}}=\sqrt{\frac{\sum \boldsymbol{v}^{\mathrm{T}} \boldsymbol{v}}{n-r}}
$$

where $n$ is the number of accepted links and $r$ is the number 
of unknowns; in this way, a small number of links with major errors can be separated from others that have a normal error distribution. To calculate the total uncertainty $\delta_{\text {rel }}$ for each station (see Table 2), the $r_{i i}$ diagonal elements of the $R$ matrix is used, with the following relationship applied:

$$
\delta_{r e l}=\sqrt{\sigma_{0}^{2} \cdot r_{i i}}
$$

Applying the procedure described above to the data acquired in 2005 for the 13 selected gravity stations, the total uncertainty is between $6 \mu \mathrm{Gal}$ and $9 \mu \mathrm{Gal}$ (see Table 2). Although the uncertainty $\delta_{\Delta g}$ between some of the pairs of stations is small enough ( $3 \mu \mathrm{Gal}$ in some cases), at the end of the compensation procedure, a larger total uncertainty at each measurement is assigned to all of the values. Consequently, the final gravity value at each station will depend both on the uncertainty $\delta_{\Delta g}$ and on the closure error of the loop formed by the stations.

\section{Hybrid gravity data}

The accuracy of the gravity measurements obtained using an absolute gravimeter is very high compared to the accuracy in measurements obtained with a relative gravimeter, although the sensitivity and complexity of the absolute gravimeters makes it very difficult to use them in any location other than under laboratory conditions. Only recently have field-usable absolute gravimeters been developed, making high-precision gravity observations possible using absolute readings [Ferguson et al. 2008]. Even though movable, the sensitivity and fragility of a field-usable absolute gravimeter complicates its use. These factors have the effect of limiting the number of points where the gravity can be measured within a specific amount of time. At present, the best way to assess the temporal and spatial gravity variations over a gravity network is to combine data collected with absolute ballistic and relative spring gravimeters in a hybrid approach [Berrino 2000, Furuya et al. 2003, D'Agostino et al. 2008, Ferguson et al. 2008].

The accuracy of relative gravimeters is known to be limited by the instrumental drift that can increase when these instruments are subjected to mechanical and thermal shocks during transportation. In addition, the measurement schedule for a relative gravity survey that occupies at least one reference point usually represents a huge amount of work for the operators. It is worth noting that if an absolute gravimeter is used at different points of a relative gravity network, many reference stations would be available, which allows loops between stations separated by very long distances to be avoided (the Mt. Etna reference stations that are not affected by volcano-related gravity changes are about $20 \mathrm{~km}$ from the summit zone). Thus the time required to accomplish discrete surveys would be drastically decreased, and the reliability of discrete data would be improved.

To reduce the inconvenience due to the use of only relative gravimeters, and to exploit instead the advantages offered by the absolute gravimeter, for the hybrid procedure,

\begin{tabular}{|c|c|c|c|c|c|}
\hline Station & Link & $\begin{array}{c}\Delta g \\
(\mathrm{mGal})\end{array}$ & $\begin{array}{c}\delta_{\Delta g} \\
(\mu \mathrm{Gal})\end{array}$ & $\begin{array}{c}g_{\mathrm{abs}} \\
(\mathrm{mGal})\end{array}$ & $\begin{array}{c}\delta_{\text {hyb }} \\
(\mu \mathrm{Gal})\end{array}$ \\
\hline IFO & ${ }^{\star}$ SLN-IFO & 9.158 & 3 & 650.837 & 5 \\
\hline MDZ & ${ }^{\star}$ GAL-MDZ & 39.736 & 4 & 640.626 & 9 \\
\hline MFO & ${ }^{\star}$ GAL-MFO & 7.570 & 2 & 608.460 & 8 \\
\hline MPA & ${ }^{\star}$ GAL-MPA & 14.894 & 3 & 585.996 & 9 \\
\hline MNZ & ${ }^{\star} \mathrm{MSC}-\mathrm{MNZ}$ & -18.120 & 5 & 615.024 & 7 \\
\hline $\mathrm{BCH}$ & ${ }^{\star} \mathrm{MSC}-\mathrm{BCH}$ & 14.009 & 7 & 647.153 & 9 \\
\hline RLN & ${ }^{\star}$ MSP-RLN & -12.868 & 5 & 681.011 & 6 \\
\hline MSP & ${ }^{\star}$ MSP-MSP & 0.062 & 4 & 693.942 & 5 \\
\hline $\mathrm{L} 81$ & ${ }^{\star}$ MSP-L81 & 16.550 & 6 & 710.430 & 7 \\
\hline MSM & ${ }^{\star}$ GPL-MSM & 25.362 & 4 & 661.430 & 6 \\
\hline GLA & ${ }^{\star}$ GPL-GLA & -31.949 & 2 & 636.067 & 5 \\
\hline GPA & ${ }^{\star} \mathrm{GPL}-\mathrm{GPA}$ & -11.231 & 5 & 656.785 & 7 \\
\hline MRO & ${ }^{\star}$ GPL-MRO & 2.281 & 6 & 670.296 & 8 \\
\hline
\end{tabular}

Table 3. Results of the hybrid gravity measurements acquired in 2010 for the 13 selected relative gravity stations and the five absolute gravity reference points of the Mt. Etna gravity network. The columns indicate, respectively, the relative stations acronym, the links between the relative stations and an absolute reference point, the gravity differences $(\Delta g)$ between them and their associated uncertainty $\left(\delta_{\Delta g}\right)$, the absolute gravity values acquired at the reference point by the FG5\#238 absolute gravimeter $\left(g_{a b s}\right)$, and the associated uncertainties $\left(\delta_{h y b}\right)$. Each relative station was linked with the closest absolute reference point, as indicated with the symbol “丸”. 
we use the absolute stations that are located on Mt. Etna volcano as references for the relative measurements acquired in the different sectors of the relative gravity network (Figure 1). In brief, we connect three or four relative gravity stations at each absolute site (used as a reference). The high-precision gravity values at all of the stations are then determined by adding the difference $(\Delta g)$ measured between each absolute station with the relative stations. All of these absolute values are transferred to $250 \mathrm{~mm}$ from the ground through the vertical gravity gradient acquired at each station, using a CG5\#8664.196 Scintrex relative gravimeter (Figure 2c). We chose this height to compare the absolute and relative measurements at the same height from the ground.

Following the above hybrid procedure, Table 3 shows the data acquired in 2010 for the western part of the volcano from the 13 relative stations (the same as those used in 2005 , to evaluate the uncertainty of the relative data; see Section 4) and at the five absolute stations located in the same area of the volcano and used as references for the relative measurements. As stated before, each relative station was linked with the closest absolute station, as labeled with the symbol * in Table 3. Following this hybrid approach, and considering that the free-air vertical gravity gradients are measured at each absolute station (Table 1), we estimate the total uncertainty at each station according to the equation:

$$
\delta_{h y b}=\sqrt{\delta_{\Delta g}^{2}+\delta_{F A G}^{2}+\delta_{a b s}^{2}}
$$

where $\delta_{\Delta g}$ is the uncertainty calculated for all of the differences $(\Delta g)$ necessary to connect a relative gravity station to an absolute site, $\delta_{F A G}$ is the uncertainty of the vertical gravity gradient, and $\delta_{a b s}$ is the total uncertainty for each absolute measurement, calculated as described in the Section 3. The uncertainties attributed to each $\Delta g\left(\delta_{\Delta g}\right)$ are calculated as described in Section 4.

The results highlight that the hybrid method that was applied to collect data in the western part of the Mt. Etna volcano includes 13 relative and five absolute stations, which allowed gravity data to be obtained with a total uncertainty, as calculated by Equation (18), of $5 \mu \mathrm{Gal}$ to $9 \mu \mathrm{Gal}$ (see Table 3). The results indicate that the final total uncertainty depends strongly on the $\delta_{\Delta g}$ uncertainty calculated between each single pair of stations, and the propagation errors are not included.

\section{Discussion and conclusions}

In this study, we compared high-precision gravity data collected at Mt. Etna volcano with different methods. As a case study, we considered first a gravity dataset that was acquired from the western side of the Mt. Etna volcano in 2005 , at 13 stations using only a relative gravimeter. The data were processed through the least-squares adjustment method, to provide gravity values and their related uncertainties. The second dataset was acquired in 2010 in the same area of the Mt. Etna volcano, and as well as the same relative gravity stations, another five absolute points were added and used as references for the relative measurements (the hybrid method). The results highlight that the uncertainty calculated with data acquired by the hybrid method is comparable with the uncertainty that affects the gravity data when a single relative gravimeter is used (see Tables 2 and 3). Obviously, this extraordinary result depends greatly on the ability of the operators to perform gravity measurements with both of these gravimeters in hostile environments. Moreover, in the latter case, the gravity values and the final total uncertainty at each station depend heavily on a compensation procedures. This means that if several wrong data are included in the analysis procedure, the final gravity values are adjusted and high uncertainty values are equally assigned to each gravity value. This provides ambiguous results and prevents an unequivocal interpretation of the anomalies observed. In addition, we must consider that the errors of the measurements increase with the number $n$ of the consecutive differences, and after the $n$-th difference, the measurement error is multiplied by $\sqrt{n}$. The final result also depends on the total number of stations included in the loop.

Conversely, the hybrid approach allows the obtaining of the gravity value at each relative station through a direct link between the absolute station and the relative station. In this case, the final total uncertainty strongly depends on the $\delta_{\Delta g}$ uncertainty that is calculated between each single pair of stations and the ambiguity in the final gravity values, which is not included due to the propagation errors during both the field measurements and the data processing.

In summary, we conclude that the hybrid approach provides the following advantages: a) it allows the optimization of the traditional techniques and strategies of gravity measurements of the Mt. Etna network, thus enabling a drastic reduction in the time required to accomplish discrete surveys and ensuring improvements to the quality of the data; b) it obtains gravity information with an accuracy that is comparable to the absolute gravity information on an extensive gravity network, which thus reduces the typical ambiguity that is inherent in the singlecomponent method; and c) it also allows the investigation of low amplitude gravity variations of the order of a few $\mu \mathrm{Gal}$, which have never been considered previously for Mt. Etna, and which can provide useful information towards our better understanding of the geophysical processes that precede and accompany volcanic phenomena.

Acknowledgements. This study was developed within the framework of the TecnoLab, the Laboratory for the Technological Advance in Volcano Geophysics, organized by INGV-CT and DIEEIUNICT. Thanks are due to the E\&P Division of the AESI Department of Eni S.p.a. for making the FG5\#238 absolute gravity meter available. 


\section{References}

Battaglia, M., P. Segall and C. Roberts (2003). The mechanics of unrest at Long Valley caldera, California. 2. Constraining the nature of the source using geodetic and micro-gravity data, J. Volcanol. Geoth. Res., 127, 219-245; doi: 10.1016/S0377-0273(03)00171-9.

Berrino, G. (2000). Combined gravimetry in the observation of volcanic processes in southern Italy, J. Geodyn., 30, 371-388.

Bonaccorso, A., A. Bonforte, G. Currenti, C. Del Negro, A. Di Stefano and F. Greco (2011). Magma storage, eruptive activity and flank instability: inferences from ground deformation and gravity changes during the 1993-2000 recharging of Mt. Etna volcano, J. Volcanol. Geoth. Res., 200, 245-254.

Bonforte, A., S. Gambino, F. Guglielmino, F. Obrizzo, M. Palano and G. Puglisi (2007). Ground deformation modeling of flank dynamics prior to the 2002 eruption of Mt. Etna, B. Volcanol., 69 (7), 757-768; doi: 10.1007/s00445006-0106-1.

Branca, S., D. Carbone and F. Greco (2003). Intrusive mechanism of the 2002 NE-Rift eruption at Mt. Etna (Italy) inferred through continuous microgravity data and volcanological evidence, Geophys. Res. Lett., 30, 2077; doi: $10.1029 / 2003$ GL018250.

Budetta, G. and D. Carbone (1997). Potential application of the Scintrex CG-3M gravimeter for monitoring volcanic activity: results of field trials on Mt. Etna, Sicily, J. Volcanol. Geoth. Res., 66, 199-214.

Budetta, G., D. Carbone and F. Greco (1999). Subsurface mass redistributions at Mount Etna (Italy) during the 1995-1996 explosive activity detected by microgravity studies, Geophys. J. Int., 138, 77-88.

Carbone, D., G. Budetta and F. Greco (2003). Bulk processes some months before the start of the 2001 Mt Etna eruption, evidenced through microgravity studies, J. Geophys. Res., 108, 2556.

Carbone, D. and F. Greco (2007). Review of microgravity observations at Mt. Etna: a powerful tool to monitor and study active volcanoes, Pure Appl. Geophys., 164, 1-22.

Chartier, J.M. (1987): Étude de l'influence de la valeur de la pression atmosphérique sur la fréquence des lasers asservis. Rapport BIPM-87/2, 19 pp.

D'Agostino, G., S. Desogus, A. Germak, C. Origlia, D. Quagliotti, G. Berrino, G. Corrado, V. d'Errico and G. Ricciardi (2008). The new IMGC-02 transportable absolute gravimeter: measurement apparatus and applications in geophysics and volcanology, Annals of Geophysics, 51 (1), 39-49.

Doebelin, E.O. (1990). Measurement System: Application and Design, 4th ed., McGraw-Hill, ISBN: 0-07-017338-9.

Ferguson, J.F., F.J. Klopping, T. Chen, J.E. Seibert, J.L. Hare and J.L. Brady (2008). The 4D microgravity method for waterflood surveillance: Part III 4D absolute microgravity surveys at Prudhoe Bay, Alaska, Geophysics, 73 (6), WA163-WA171; doi: 10.1190/1.2992510.

Furuya, M., S. Okubo, S. Sun, W. Tanaka, Y. Oikawa, J. Watanabe and H.T. Maekawa (2003). Spatiotemporal gravity changes at Miyakejima Volcano, Japan: caldera collapse, explosive eruptions and magma movement, J. Geophys. Res., 108; doi: 10.1029/2002JB001989.

Greco, F., G. Currenti, C. Del Negro, R. Napoli, G. Budetta, M. Fedi and E. Boschi (2010). Space-time gravity variations to look deep into the southern flank of Etna volcano, J. Geophys. Res., 115, B11411; doi: 10.1029/2009JB 006835.

Hautmann, S., J. Gottsmann, A.G. Camacho, N. Fournier, I.S. Sacks, R. Stephen and J. Sparks (2010), Mass variations in response to magmatic stress changes at Soufrire Hills Volcano, Montserrat (W.I.): insights from 4-D gravity data, Earth Planet. Sc. Lett., 290 (1-2), 83-89.

Niebauer, T.M., G.S. Sasegawa, J.E. Faller, R. Hilt and F. Klopping (1995). A new generation of absolute gravimeters, Metrologia, 32, 159-180.

Van Camp, M., S.D.P. Williams and O. Francis (2005). Uncertainty of absolute gravity measurements, J. Geophys. Res., 110, B05406; doi: 10.1029/2004JB003497.

Warburton, R. and J. Goodkind (1977). The influence of barometric pressure variations on gravity, Geophys. J. Roy. Astr. S., 48 (3), 281-292.

Watermann, H. (1957). Über systematische Fehler bei Gravimetermessungen. Deutsche Geodätische Kommission, DGK C21, München.

Williams-Jones, G., and H. Rymer (2002). Detecting volcanic eruption precursors: a new method using gravity and deformation measurements, J. Volcanol. Geoth. Res., 113, 379-389; doi: 10.1016/S0377-0273(01)00272-4.

\footnotetext{
${ }^{\star}$ Corresponding author: Antonio Pistorio, Istituto Nazionale di Geofisica e Vulcanologia, Sezione di Catania, Osservatorio Etneo, Catania, Italy; email: antonio.pistorio@ct.ingv.it.

C 2011 by the Istituto Nazionale di Geofisica e Vulcanologia. All rights reserved.
} 